\title{
Characterizing protein protonation microstates using Monte Carlo sampling
}

\author{
Umesh Khaniya ${ }^{1,2}$, Junjun Mao ${ }^{1}$, Rongmei Wei ${ }^{1,3}$, M. R. Gunner ${ }^{1,2,3^{*}}$
}

${ }^{1}$ Department of Physics, City College of New York, New York 10031, United States

${ }^{2}$ Department of Physics, The Graduate Center, City University of New York, New York 10016

${ }^{3}$ Department of Chemistry, The Graduate Center, City University of New York, New York 10016

* Correspondence: Department of Physics, City College of New York, 160 Convent Avenue, New York, NY 10031, USA

E-mail address: mgunner@ccny.cuny.edu (M.R. Gunner) 


\begin{abstract}
:
Proteins are polyelectrolytes with acidic or basic amino acids making up $\approx 25 \%$ of the residues. The protonation state of all Asp, Glu, Arg, Lys, His and other protonatable residues, cofactors and ligands define each protonation microstate. As all of these residues will not be fully ionized or neutral, proteins exist in a mixture of microstates. The microstate distribution changes with $\mathrm{pH}$. As the protein environment modifies the proton affinity of each site the distribution may also change in different reaction intermediates or as ligands are bound. Particular protonation microstates may be required for function, while others exist simply because there are many states with similar energy. Here, the protonation microstates generated in Monte Carlo sampling in MCCE are characterized in HEW lysozyme as a function of $\mathrm{pH}$ and bacterial photosynthetic reaction centers (RCs) in different reaction intermediates. The lowest energy and highest probability microstates are compared. The $\Delta \mathrm{G}, \Delta \mathrm{H}$ and $\Delta \mathrm{S}$ between the four protonation states of Glu35 and Asp52 in lysozyme are shown to be calculated with reasonable precision. A weighted Pearson correlation analysis identifies coupling between residue protonation states in RCs and how they change when the quinone in the $\mathrm{Q}_{\mathrm{B}}$ site is reduced.
\end{abstract}




\section{INTRODUCTION}

Proteins are large dynamic molecules that move amongst many conformations as the atoms change their positions. With $\mathrm{N}$ atoms there are $3 \mathrm{~N}-6$ vibrations ranging from high-frequency vibrations of individual bonds to larger, slower breathing modes of the protein as a whole. Positional changes can be required for function, and they are also inevitable given the low barriers for many motions. Conformational changes modify hydrogen bond patterns, expose buried charged side chains to the solution, and stabilize bound ligands.

Protons are sometimes overlooked reactants in many chemical reactions. They are lost as chemical bonds are made and many redox reactions are coupled to proton binding. The transmembrane electrochemical gradient, including a $\Delta \mathrm{pH}$, provides an essential store of cellular energy. Protons are therefore routinely transferred in and out of protein active sites and across membrane embedded proteins, requiring changes in protonation states along proton transfer pathways as well as at active sites. Drug binding has been shown to modify the protonation states of the protein as well as the drug itself. ${ }^{1-3}$ Ion binding can be coupled to changes in protonation states. ${ }^{4,5}$

It has been underappreciated that proteins exist in a distribution of protonation states. An average protein has approximately $25 \%$ acidic and basic residues. ${ }^{6}$ A protonation microstate identifies the protonation state of all acidic and basic groups in the molecule. For $\mathrm{N}$ titratable groups there are $2^{\mathrm{N}}$ protonation microstates. Tautomers are microstate with the same net charge but with the protons shifting position. The total number of tautomers for $\mathrm{m}$ protons distributed over $\mathrm{N}$ binding sites is

$$
\frac{\mathrm{N} !}{m !(N-m) !}
$$

Distributions of protonation microstates occur when residue $\mathrm{pK}_{\mathrm{a}} \mathrm{s}$ are near the $\mathrm{pH}$ in any individual structure. Also, the ensemble of protein conformations generates an additional ensemble of protonation microstates since the proton affinity of residues will shift as they sample different environments. Protonation states also change when ligands are bound ${ }^{2,3}$ or when redox reactions occur at bound cofactors ${ }^{7,8}$. Proton pumps move protons through membrane embedded proteins as changes in protein conformation and redox states modify residue proton affinity ${ }^{9-13}$. As with the protein conformational states, some protonation microstates are essential for protein function while others simply result from multiple microstates being close in energy.

Analysis of microstates. The ensembles of states generated in any simulation method is limited by the degrees of freedom and the methods of moving between states. In Molecular Dynamics (MD) trajectories, atoms and molecules move in time steps, propelled by classical molecular mechanics forces. In standard MD simulations, molecules remain in a single chemical state so their protonation state is fixed at the beginning. Each time step represents a microstate of the system. In Monte Carlo (MC) sampling discrete states are generated based on the chosen degrees of freedom and then retained or rejected based on their energy. States sampled by MC can differ by changing atomic positions, number of atoms or chemical state including protonation or redox state. A microstate identifies one choice for all degrees of freedom.

MD trajectories yield large data sets with the molecule moving between many conformations. The challenge is to extract the biological significance of the data. A fraction of conformational microstates (frames), of the order of one in a thousand, are saved for analysis. Clustering algorithms group frames to cover the range of diverse conformations based on the similarity score of investigator chosen features. Well defined clustering approaches include hierarchical clustering (linkage based) ${ }^{14,15}$, center-based (K-means, K-centers) clustering ${ }^{14,16}$. 
We will describe here analysis of protonation microstates obtained with the MCCE program. It uses MC sampling to generate an ensemble of residue and ligand protonation, conformation and redox states ${ }^{17}$. This is one of a group of programs that calculate the ensemble of protonation states for a protein using Grand Canonical Monte Carlo sampling. They find the probability of each residue being protonated at a given proton chemical potential $(\mathrm{pH})$. Methods include those using semiempirical force fields ${ }^{18,19}$ or Continuum Electrostatics. ${ }^{20,21}$ They can have fixed protein positions ${ }^{21,22}$, include sampling side chain positions with a rigid backbone as in MCCE or as in cpHMD, be embedded in full MD. ${ }^{23-27}$ cpHMD usually uses a classical electrostatic force field with a vacuum dielectric constant, but can also use a polarizable force field to obtain a better treatment of the protein dielectric response ${ }^{28}$. Available software packages for $\mathrm{pK}_{\mathrm{a}}$ prediction include MCCE ${ }^{17}, \mathrm{PROPKA}^{29}$, DelPhiPka ${ }^{30}, \mathrm{H}^{++31}, \mathrm{PypKa}^{32}$

Monte Carlo sampling requires consideration of millions of randomly chosen microstates. Traditional calculation of protonation using MC compresses the output to provide only the average probability for the protonation state of each residue at a given $\mathrm{pH}$. A titration is simulated by carrying out the calculation at multiple $\mathrm{pHs}$ to derive a $\mathrm{pK}_{\mathrm{a}} \mathrm{s}$ for all residues. If other degrees of freedom, such as ligand binding or residue conformation are allowed, their probabilities are also found. However, the full microstate distribution contains additional information such as the range of protein net charge and the correlation between protonation of individual sites and with any other available degrees of freedom. Microstate analysis can find the probability of higher energy states that may be reaction intermediates. The protonation microstates can also provide a complete assignment of protonation states for all residues and tautomer states for all His as input for MD. The microstates with the lowest energy or highest probability can be compared.

Here we will describe using Grand Canonical Monte Carlo (GGMC) within MCCE to characterize the distribution of protonation microstates and their thermodynamic properties using hen white lysozyme and $R b$. sphaeroides photosynthetic reaction centers (RCs) as examples. Lysozyme has been used as a test case for $\mathrm{pK}_{\mathrm{a}}$ predications and the protonation probability as a function of $\mathrm{pH}$ has been measured. ${ }^{33-36} \mathrm{RCs}$ have a complex network of protonatable residues that function to modulate the electrochemistry of quinone reduction and to serve as a proton transfer pathway to the quinone. ${ }^{37,38}$ This larger system will show the complexity of protonation microstates in a system that requires proton transfer for function. The microstate energy distribution, the distribution of microstates with unique charges, the thermodynamics of an individual protonation reaction and the correlation of the protonation of individual residues will be described.

\section{METHODS}

The Boltzmann distribution of protonation states and side chain polar proton positions are found for hen egg-white Lysozyme (PDB ID: 4lZT ${ }^{39}$ ) and the reaction centers (RCs) from the purple non-sulfur photosynthetic bacteria $R b$. Sphaeroides (PDB ID: $1 \mathrm{AIG}^{40}$ ) using the MCCE $\operatorname{program}^{17}$.

Conformer generation determines the degrees of freedom. In MCCE the protein backbone is always fixed. Residue side chains and ligands can be given multiple choices that define their protonation and conformational state. Each choice is a 'conformer'. While MCCE can carry out full side chain rotamer sampling, 'isosteric' conformers are used here for simplicity ${ }^{41}$. MCCE can also include explicit waters with multiple positions. ${ }^{42}$ However as is customary, all water molecules in the crystal structure are deleted and replaced by implicit solvent in lysozyme while 
28 crystallographic waters are retaining in the RCs. All protonatable residues (Glu, Asp, Arg, Lys, His, Tyr and Cys and N- and C-termini) have conformers that are charged and others that are neutral. Side chain conformers are made that differ in the position of polar side chain protons. This includes conformers with different neutral His tautomers, Asn and Gln amide termini orientation and the position of hydroxyl protons on Tyr, Ser, Thr and Cys and proton positions on neutral Asp and Glu. The ubiquinone in RCs can be oxidized, Q, or anionic semiquinone, Q ${ }^{-}$. Each residue has from one to six conformers that are selected in MC sampling.

MCCE force field. A microstate of the protein is one selected conformer for each residue. The energy of each microstate is divided into a reference energy, and self and pairwise terms. ${ }^{17}$ The energy of any microstate is obtained from the pre-calculated energy lookup table. The energy $\left(\Delta \mathrm{H}^{\mathrm{x}}\right)$ of microstate $\mathrm{x}$ is:

$$
\begin{aligned}
\Delta H^{x}= & \sum_{i=1}^{M} \delta_{x, i}\left\{\left[2.3 m_{i} k_{b} T\left(p H-p K_{\text {sol, },}\right)+n_{i} F\left(E_{h}-E_{m \text { sol }, i}\right)\right]\right. \\
& +\left(\Delta \Delta G_{r x n, i}+\Delta \Delta G_{b k b n, i}^{C E}+\Delta \Delta G_{b k b n, i}^{L J}+\Delta \Delta G_{\text {torsion }, i}+\Delta \Delta G_{S A S, i}\right) \\
& \left.+\sum_{j=i+1}^{M} \delta_{x, j}\left[\Delta G_{i j}^{C E}+\Delta G_{i j}^{L J}\right]\right\}
\end{aligned}
$$

The first line describes the energy for each residue type to be in its protonated or unprotonated form or to be oxidized or reduced in solution. Lines two and three will describe how the protein shifts the proton and electron affinity of the conformer. $\mathrm{M}$ is the total number of conformers. $\delta_{\mathrm{x}, \mathrm{i}}$ is 1 if conformer $\mathrm{i}$ is present in microstate $\mathrm{x}$ or 0 otherwise. $\mathrm{m}_{\mathrm{i}}$ is 1 for basic protonated, -1 for acidic deprotonated and 0 for all neutral conformers. $\mathrm{pK}_{\text {sol, }, \mathrm{i}}$ is the reference $\mathrm{pK}_{\mathrm{a}}$ of this residue type in solution, $\mathrm{E}_{\mathrm{m}}$ is the electrochemical midpoint potential of this redox active group. $\mathrm{F}$ is the Faraday constant, $\mathrm{n}_{\mathrm{i}}$ is the change in the number of electrons on a conformer type that is taking part in a redox titration. $\mathrm{pH}$ and $\mathrm{E}_{\mathrm{h}}$ are the relevant solution parameters describing the chemical potential of protons or electrons that will come to equilibrium with the protein.

The second line of equation 2 describes the self-energies of the conformer that form the microstates. These energies are independent of the conformer choice for other residues. These are: the loss of conformer solvation energy as it is moved from solution to its position in the protein, torsion energy, continuum electrostatic (CE) and Lennard Jones (LJ) van der Waals interactions with the fixed backbone amides and favorable, van der Waals interactions of the exposed side chain surface with the implicit solvent. The third line gives the continuum electrostatic and Lennard Jones pairwise interactions between each pair of conformers in other residues. All energies are calculated prior to $\mathrm{MC}$ sampling. Electrostatic energies are calculated using the Poisson-Boltzmann solver in Delphi ${ }^{20,43,44}$ using Parse charges ${ }^{45}$, a dielectric constant of 4 for protein and 80 for solvent with an implicit salt concentration of $150 \mathrm{mM}$. MCCE corrects the continuum electrostatic energies for the changes in the dielectric boundary due to changes in surface rotamers. ${ }^{17}$ Non-electrostatic energies use the Amber force field. ${ }^{46}$

Monte Carlos sampling. In MCCE microstates change by random choices, first of a residue then of a conformer available for that residue. If the chosen residue contains conformers which interact with an absolute value $>0.5 \mathrm{kcal} / \mathrm{mol}$ with conformers of another residue, additional conformer changes for that other residue will be made $50 \%$ of the time. Here conformers in several residues switch, followed by a single decision using the Metropolis-Hastings algorithm to accept or reject the change. Allowing one to three residues to change helps avoid electrostatic or van der Waals clashes between strongly coupled groups and speeds convergence. ${ }^{47,48}$

The Monte Carlo sampling routine starts with a random microstate with one conformer assigned to each residue. The program then goes through annealing, conformer reduction, and MC 
sampling. Annealing changes the acceptances temperature in the Metropolis-Hastings acceptance criteria. MC sampling is then carried out at room temperature. By default, equilibration samples $3000 \mathrm{MC}$ steps/conformer for lysozyme and 300 for RCs. Prior to the production phase, conformers that are rarely chosen are removed from the list of sampled conformers. The default cut-off used here, is that a conformer with a probability less than 0.001 is discarded. This speeds convergence and results in acceptance of MC steps of $\approx 30 \%$. However, the early elimination of conformers creates problems in highly correlated systems so it can be modified as needed. ${ }^{42}$ The discarded conformers are excluded from all later sampling. "Free residues" have a choice of conformers. A residue whose protonation state becomes fixed by all conformers of one protonation type being discarded may still sample conformers with different positions. If the probability of a single conformer for a residue is unity then that is a fixed residue, which is removed from the sampling list.

The energy for the full ensemble is calculated once for the first random microstate. In each subsequent step the interactions with the conformer chosen for change is subtracted from the ensemble energy and the energy of the new conformer for that residue is added back, speeding the energy calculation in each MC step. The default number of Monte Carlo steps for the production phase, which microstates will be recorded, is $2000 *$ number of free conformers. Six independent Monte Carlo cycles of this length starting with a random microstate formed from free conformers are carried out. The six runs are combined, but they can be compared to check the quality of Monte Carlo convergence.

Microstate information storage. A major challenge is to record the several million accepted microstates in a readable form. The MCCE algorithm has several features that make this easier. One is that residue conformers are premade and discrete so that we can use the conformer indices as a unique identifier for each microstate. Also, only a few residues are changed on each step so that we need only record the conformers that have changed when a microstate is accepted. Also, no dynamic information needs to be recorded for fixed residues as their conformer never changes.

The microstate file starts with making a list of conformer IDs that are removed from sampling because they had low probability during pre-production MC sampling and the list of conformers that will be sampled. Likewise, a list of the fixed residues with only one available conformer is separated from the list of free residues which have multiple available conformers. The conformers for the fixed residues are saved at the start. The first line of each $\mathrm{MC}$ run gives the initial microstate, listing the occupied conformer ID for all free residues. Subsequent lines record only the conformer IDs that have changed in this step, the microstate energy $(\mathrm{kcal} / \mathrm{mol})$ and the microstate count, which is the number of microstates that are rejected before the next, new microstate is accepted. Links to Jupiter notebooks with tools and tutorials for microstate analysis are found in SI Link S1.

Generating the protonation microstate ensemble. The microstate file is a ticker-tape that must be replayed to find the subsequent microstates. The information can be sorted on many properties. Here we will describe how protonation microstates are identified. Many conformational microstates are aggregated for each unique protonation microstate.

First, the charge of fixed residues that have only a single conformer available in MC is determined. This charge is summed and treated as the background charge which will be added to find the total protein charge in each microstate.

Then the ticker-tape is read to place all microstates of free residues into memory. The microstate file gives the conformer ID of each free residue. A microstate is constructed with only 
the charge of this conformer. This is a vector of the length of all free residues with an entry of -1 , 0 or 1 (though groups with other charge states are allowed). Then we determine which charge microstates are unique. The length of the unique microstate vectors is reduced to contain only residues of interest, here acidic and basic residues and the ubiquinone. At each stage the MC count is retained and is summed when multiple microstates are grouped into one category.

Correlation between microstates. Correlation measures the strength of the relationship between two variables with the sign giving the direction of the trend. The weighted Pearson Correlation $\left(\mathrm{r}_{\mathrm{pq}}\right)^{49}$, used here to find residues whose protonation changes are coupled together, is given by:

$$
r_{p q}=\frac{\sum_{i=1}^{n} w_{i}\left(p_{i}-\bar{p}\right)\left(q_{i}-\bar{q}\right)}{\sqrt{\sum_{i=1}^{n}\left(w_{i}\left(p_{i}-\bar{p}\right)^{2}\right) \sum_{i=1}^{n}\left(w_{i}\left(q_{i}-\bar{q}\right)\right)^{2}}}
$$

Here $w_{i}$ is the weight, $\mathrm{p}_{\mathrm{i}}$ and $\mathrm{q}_{\mathrm{i}}$ are the protonation states for two residues in a microstate $\mathrm{i}, \bar{p}$ and $\bar{q}$ are the mean value of $p_{i}$ and $q_{i}$ respectively and $n$ is the number of unique accepted charge microstates. The unique charge file is the input. Only those residues whose protonation states take different values in the ensemble are included. The weight of each microstate is the count of accepted microstates that have this unique protonation state. $r_{p q}$ is calculated for all pairs of residues with variable charge.

\section{RESEULTS AND DISCUSSION}

Traditionally MCCE analysis provides the Boltzmann averaged protonation and side chain and ligand position for a protein ${ }^{41}$ or other macromolecules. ${ }^{50,51}$ The goal is to now characterize the distribution of protonation microstates in the Boltzmann distribution. In MCCE a microstate defines both residue and ligand charge and position. Protonation microstates, which define the charge of every acidic and basic residue, will exist in many conformational states. The charge state identifies the net, total charge in the microstate. Tautomers are groups of protonation microstates that have the same charge but with the protons distributed over different residues.

Microstate Energy distribution for lysozyme. Lysozyme is a small protein with 129 residues that is a benchmark protein for $\mathrm{pK}_{\mathrm{a}}$ calculations. ${ }^{17}$ The seven Asp, two Glu, one His, six Lys and N- and C-termini are assigned ionized and neutral conformers. The isosteric conformer routine without explicit waters in MCCE created 284 conformers. Approximately 1 to 1.5 million microstates are sampled. Considering both protonation and conformation degrees of freedom $94 \%$ of the accepted microstates in Monte Carlo sampling are unique, meaning they are not revisiting a previously accepted microstate.

Figure 1 shows the distribution of microstate energies in the Boltzmann distribution. Even this small protein, there is a significant energy range. The full width at half maximum (FWHM) of the skewed normal distribution is $3.61 \mathrm{kcal} / \mathrm{mol}$. There are a small number of microstates at lowest energy, which are well separated from those at highest probability. The shape of the probability distribution is similar at all $\mathrm{pHs}$ and for small proteins such as lysozyme and large proteins such as RCs (Figure S1). 


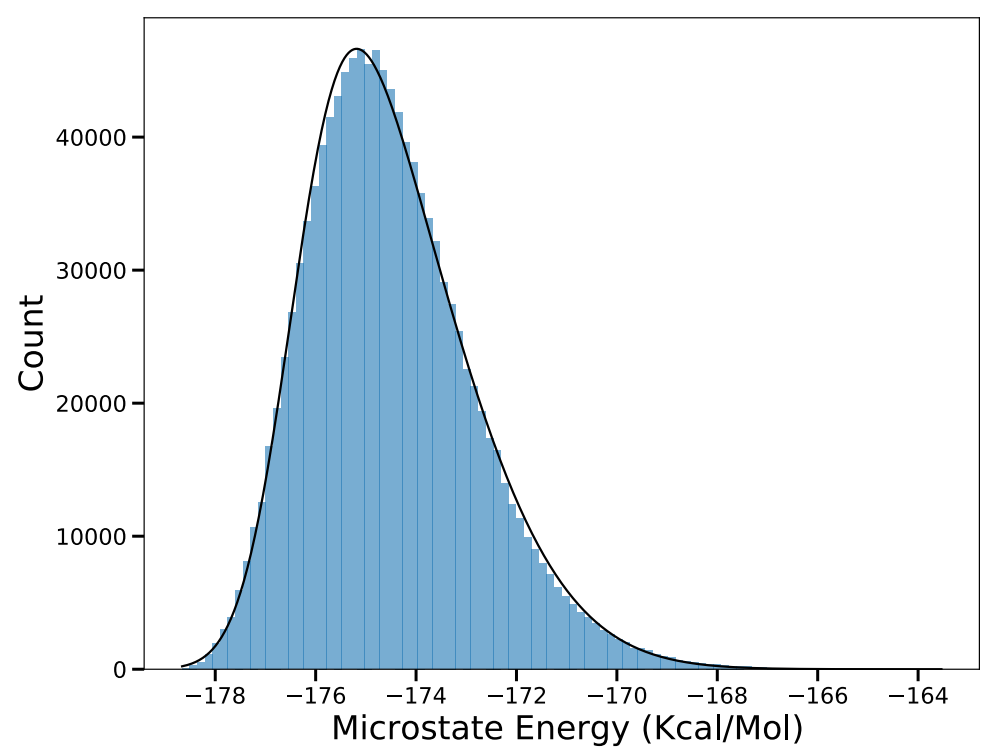

Figure 1. Microstate Energy distribution for lysozyme (PDB ID: 4LZT) at pH 7. The count is the number of times microstates in that energy window are accepted. Black line is best fit to a skewed normal distribution curve with skew of 2.86 .

In the lysozyme calculation, at $\mathrm{pH} 4$ there are $>500,000$ unique accepted conformation/protonation microstates and $>200,000$ at $\mathrm{pH} 7$. Near the residue $\mathrm{pK}_{\mathrm{a}}$ both protonated and deprotonated conformers are in the accepted ensemble. Thus, the number of unique accepted microstates reflects the number of residues that are titrating (Table 1). In lysozyme most residues have $\mathrm{pK}_{\mathrm{a}} \mathrm{s}$ close to their solution values. Thus, at low $\mathrm{pH}$ the acids are titrating. At $\mathrm{pH} 4$ ten residues have significant probability of being either charged or neutral leading to 222 unique protonation microstates. Near $\mathrm{pH} 7$ most Asp and Glu and the C-terminus are stably deprotonated and Lys and Arg protonated. Here, the protonation microstates reflect the mixture of His and Nterminus protonation states (Table S1). At $\mathrm{pH} 7$ only six residues are in a distribution of protonation states and there are only 24 different protonation microstates in the accepted ensemble (Table 1).

\begin{tabular}{|c|c|c|c|c|c|c|c|}
\hline & \multicolumn{2}{|c|}{ Number: All microstates } & \multicolumn{4}{|c|}{ Number: Protonation microstates } & \multirow{2}{*}{$\begin{array}{c}\text { Number: } \\
\text { Residues } \\
\text { Change } \\
\text { protonation }\end{array}$} \\
\hline & MC steps & $\begin{array}{c}\text { Unique } \\
\text { accepted }(\%)\end{array}$ & Unique & $\begin{array}{l}\text { Lowest } \\
\text { energy* }\end{array}$ & $\begin{array}{l}\text { Average } \\
\text { energy* }\end{array}$ & $\begin{array}{l}\text { Highest } \\
\text { energy* }\end{array}$ & \\
\hline $\mathrm{pH}$ & \multicolumn{7}{|c|}{ Lysozyme } \\
\hline 4 & $1,500,000$ & $\begin{array}{l}527,014 \\
(95.4 \%)\end{array}$ & 222 & 4 & 89 & 6 & 10 \\
\hline 5 & $1,500,000$ & $\begin{array}{c}484,152 \\
(94.59 \%)\end{array}$ & 147 & 2 & 28 & 2 & 11 \\
\hline 6 & $1,200,000$ & $\begin{array}{c}430,904 \\
(94.28 \%)\end{array}$ & 38 & 2 & 15 & 7 & 7 \\
\hline 7 & $1,200,000$ & $\begin{array}{c}447,522 \\
(94.72 \%) \\
\end{array}$ & 24 & 4 & 16 & 4 & 6 \\
\hline State & \multicolumn{7}{|c|}{$\mathrm{RCs}(\mathrm{pH} 7)$} \\
\hline $\mathrm{Q}_{\mathrm{B}}$ & $8,700,000$ & $\begin{array}{c}2,735,799 \\
(99.19 \%)\end{array}$ & 60,381 & 3 & 24,254 & 1 & 53 \\
\hline $50: 50$ & $8,294,680$ & $\begin{array}{l}2,765,120 \\
(99.19 \%)\end{array}$ & 70,713 & 4 & 27,775 & 6 & 51 \\
\hline
\end{tabular}




\begin{tabular}{|c|c|c|c|c|c|c|c|}
\hline $\mathrm{Q}_{\mathrm{B}}^{\bullet^{-}}$ & $8,700,000$ & $\begin{array}{c}2,720,450 \\
(99.21 \%)\end{array}$ & 54,448 & 5 & 22,202 & 3 & 48 \\
\hline
\end{tabular}

Table 1. Characterization of accepted microstates obtained with Metropolis-Hastings sampling. The MC steps are the aggregate of 2,000 times the number of free conformers times six restarts. Unique microstates have different protonation and or conformation. Protonation microstates differ in location of protons on acidic and basic groups. *Number of unique protonation states within $1.36 \mathrm{kcal} / \mathrm{mol}$ of the lowest or highest energy or of $\pm 0.68 \mathrm{kcal} / \mathrm{mol}$ of the average energy. There are 27 protonatable residues (Asp, Glu, Arg, His and Lys) and chain termini in lysozyme and 132 in RCs. The number of residues that change protonation have different charge states in the microstate ensemble. MC sampling for RCs is carried out with the ubiquinone in the $\mathrm{Q}_{\mathrm{B}}$ site being the neutral quinone, $\mathrm{Q}_{\mathrm{B}}$, the anionic semiquinone $\mathrm{Q}_{\mathrm{B}}{ }^{-}$or with the $\mathrm{E}_{\mathrm{h}}$ at the $\mathrm{E}_{\mathrm{m}}$ for the quinone so there is a 50:50 mixture of the two states.
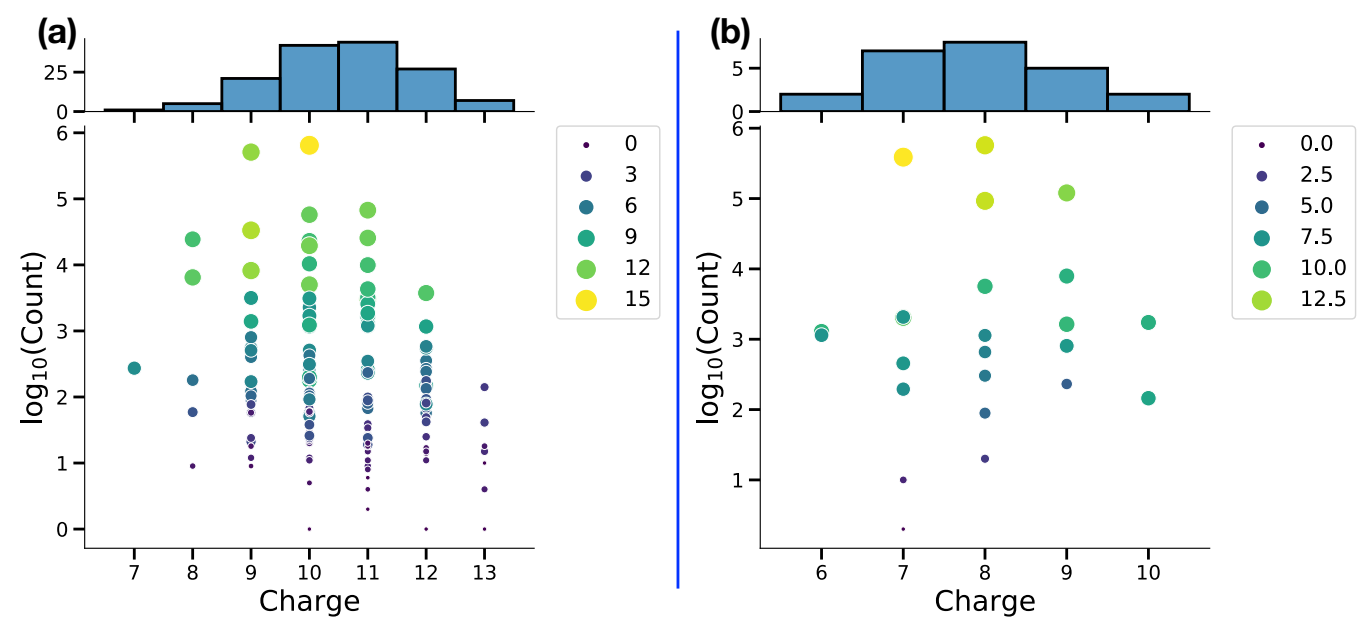

Figure 2. Unique tautomer charge distribution for lysozyme at $\mathrm{pH}$ (A) 5 and (B) 7. Each point in the scatter plot is a unique protonation microstate. The count gives its acceptance in MC sampling, with high count indicating higher probability. Color and size of points correspond to the range of microstate energies associated with all microstates found for that protonation microstate. Histogram at the top shows the total number of unique protonation microstates with a given charge. Thus, it counts the number of tautomers at each charge.

The distribution of charge and tautomer states. Figure 2 shows the distribution of the unique accepted protonation microstates. This mirrors the distribution of charge states that can be found experimentally. However, experimental measurements of protein net charge combine the charge of the protein and of bound ions ${ }^{52-54}$. There are 147 unique protonation states for lysozyme at $\mathrm{pH} 5$ and 24 at $\mathrm{pH}$ 7. The ensemble average charge is 9.68 at $\mathrm{pH} 5$ and 7.78 at $\mathrm{pH} 7$ (Figure $\mathrm{S} 2$ ). At $\mathrm{pH} 5$ proteins with charge ranging from 7 to 13 are found, though those with charge 9 or 10 are most probable, having the highest count. Each vertical line of dots is a group of tautomeric states, with the same charge but different proton distributions. In MCCE every unique charge microstate exists in a many tautomer and conformational microstates covering a wide energy range (Figure S3). Larger and more yellow dots in Figure 2 have more underlying conformational states with this distribution of protons.

Even a system as small as lysozyme can have a significant number of unique protonation microstates. However, only a small number are found with significant probability in the Boltzmann ensemble. Thus, there are $2^{18}$ possible protonation states, but at $\mathrm{pH} 7$ only 6 residues have different 
protonation states in the microstate ensemble. However, of the $2^{6}(64)$ possible states only 24 are ever in an accepted microstate. Figure 2 shows there are two microstates at either $\mathrm{pH} 5$ or 7 that have several fold more occupancy in MC sampling than any others. Table S1 lists all 24 accepted protonation microstates for lysozyme at $\mathrm{pH} 7$ as an example. Only three microstates have a probability greater than $10 \%$ and they sum to $90 \%$ of the ensemble. At $\mathrm{pH} 5,7$ states sum to a probability of $90 \%$.

Thermodynamic parameters for protonation reactions from analysis of the microstate ensemble. The changes in the free energy of a reaction determines the proportion of reactants and products at the system at equilibrium $\left(\mathrm{K}_{\mathrm{eq}}\right)$. The changes in enthalpy and entropy give insight into the nature of what changes in the reaction. Measurements of the equilibrium constant, $\mathrm{K}_{\mathrm{eq}}, \Delta \mathrm{H}$ and $\Delta \mathrm{S}$ are common, and there are methods to calculate these parameters from MD trajectories. ${ }^{55-57}$ However, calculating $\Delta \mathrm{G}, \Delta \mathrm{H}$ and especially $\Delta \mathrm{S}$ for complex biological molecules often leads to large uncertainty as it can be hard to sample enough states. ${ }^{58-62}$ Here we analyze the accepted microstates in MCCE to determine the thermodynamic properties of the coupled protonation reactions of Glu35 and Asp52 in lysozyme (Figure 3).

To determine the thermodynamic variables, the MC microstates are divided into four groups, each with a different protonation state for the two residues. The enthalpy is the average energy for the ensemble of microstates with appropriate protonation for the two acids:

$\Delta \mathrm{H}=$ Average (microstate energy $\mathrm{x}$ count microstate product) - Average (microstate energy x count microstate reactant)

The change in free energy between pairs of states is:

Therefore, entropy is:

$$
\Delta \mathrm{G}=-\mathrm{RT} \ln \frac{\text { Count }_{\text {product }}}{\text { Count }_{\text {reactant }}}
$$

$$
\mathrm{T} \Delta \mathrm{S}=\Delta \mathrm{H}-\Delta \mathrm{G}
$$

Additional $\mathrm{MC}$ sampling reduces the standard deviation for the calculation of $\Delta \mathrm{S}$. Here the number of MC steps for annealing and for the production phase are increased 10-fold, with 20,000 steps/conformer carried out with the sampling of the free conformers restarted with a new randomly chosen microstate six times.

Figure 3 shows the results for one MCCE calculation at $\mathrm{pH} 4$ where the system is in a mixture of the four protonation microstates. The results for the reaction starting from both acids neutral to both ionized was calculated for 10 independent $\mathrm{MC}$ calculations gives values of $\Delta \mathrm{G}$ of $0.95 \pm 0.16 \mathrm{kcal} / \mathrm{mol}$ with a $\Delta \mathrm{H}$ of $-0.35 \pm 0.02$ and $-\mathrm{T} \Delta \mathrm{S}$ of $1.30 \pm 0.02 \mathrm{kcal} / \mathrm{mol}$. Thus, within the constraints of the limited degrees of freedom of the MCCE calculation thermodynamic variables can be derived from the Boltzmann ensemble with reasonable precision.

Examination of the thermodynamic square shows that the state with Glu35 neutral and Asp52 ionized is favored. This agrees with the ensemble average probability where Glu35 is $8 \%$ ionized and Asp52 is $78 \%$ ionized. The breakdown of $\Delta \mathrm{H}$ and $\Delta \mathrm{S}$ shows that $\Delta \mathrm{H}$ plays the larger role, favoring this state. 


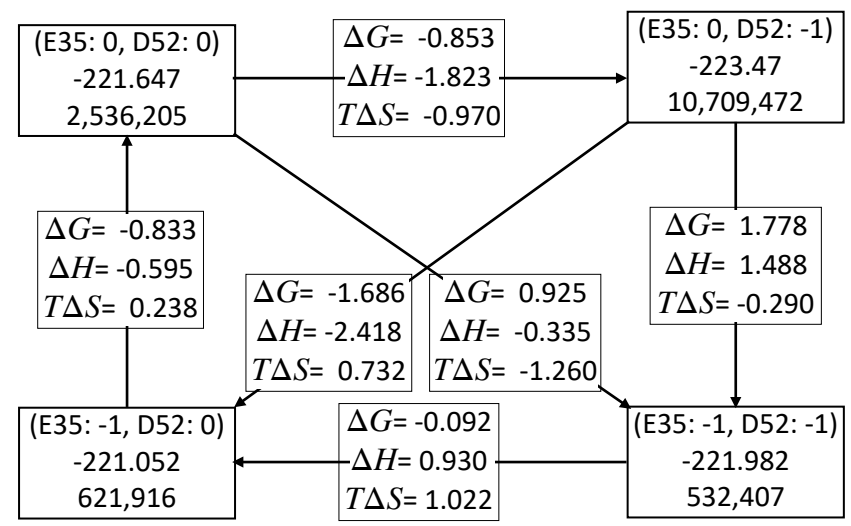

Figure 3. Thermodynamic box for titration of Glu35 and Asp52 in lysozyme at pH 4 at $298.15 \mathrm{~K}$. The full microstate ensemble is sorted into the four protonation states of these two residues and these groups are independently characterized. Corner boxes: first row is charge of Glu35 and Asp52; second row is the average microstate energy $(\mathrm{H})$; third row is the count of microstates in this protonation state. The boxes along the arrows give the $\Delta \mathrm{G}, \Delta \mathrm{H}$ and $\mathrm{T} \Delta \mathrm{S}$ for the transition between different protonation states. The top right and bottom left states are tautomers. All energies are in $\mathrm{kcal} / \mathrm{mol}$.

Thermodynamic properties with change in temperature. The $\Delta \mathrm{S}$ is traditionally measured from the temperature dependence of a reaction. The same can be done here. The same energy look-up tables are used, but the temperature of $270 \mathrm{~K}, 298.15 \mathrm{~K}, 320 \mathrm{~K}$ and $340 \mathrm{~K}$ is used for the Metropolis-Hastings test for microstate acceptance. Ten independent MC calculations are carried out at each temperature. All calculated $\Delta \mathrm{G}, \Delta \mathrm{H}$ and $\Delta \mathrm{S}$ for the system are provided in the Table S3B-D. The standard deviation of the thermodynamic variables is $<0.2 \mathrm{kcal} / \mathrm{mol}$, showing the MCCE microstate ensemble can provide reproducible values even for the estimate of $\Delta \mathrm{S}$. The same values are obtained from the Van't Hoff plot of $\mathrm{K}_{\mathrm{eq}} \mathrm{vs} 1 / \mathrm{T} .{ }^{59}$ The reaction is exothermic so heat would be released on ionization of the two acids. The standard deviation of the $\Delta \mathrm{G}$ or $\mathrm{K}_{\mathrm{eq}}$ is larger at lower temperatures.
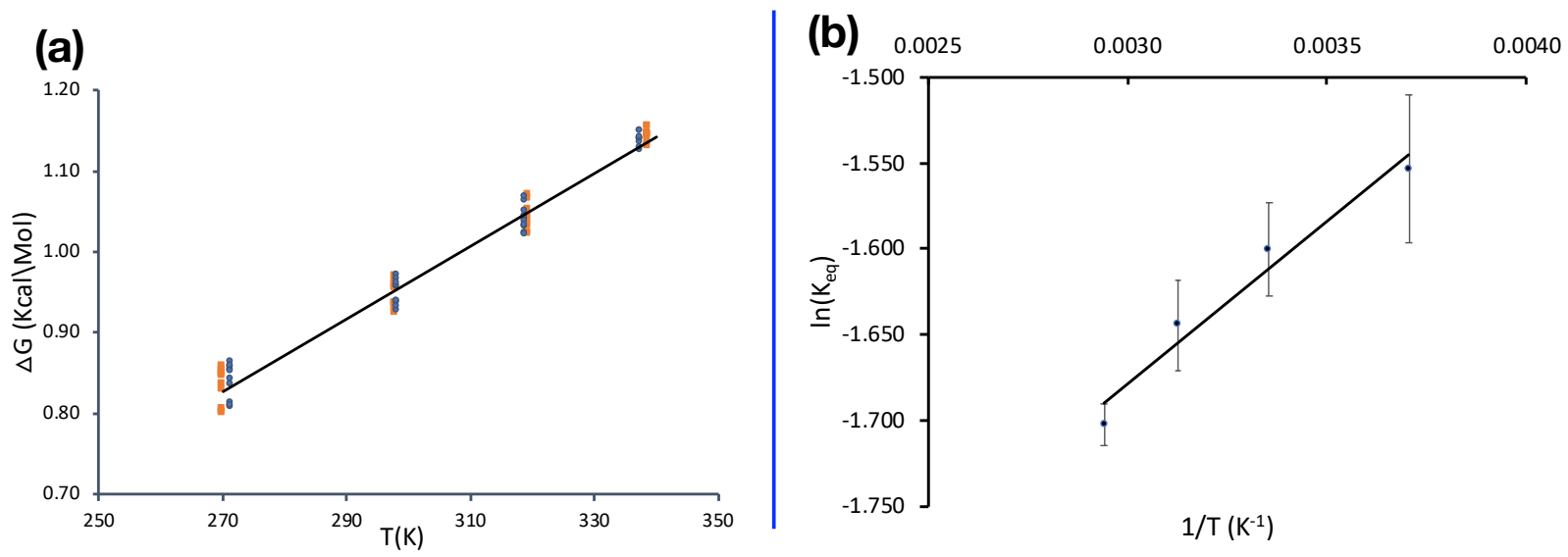

Figure 4. Temperature dependence of thermodynamic parameters. (A) Change in $\Delta G$ for the reaction taking Glu35 and Asp52 with both neutral to both ionized with temperature. Line is the linear best fit and each dot represent the $\Delta G$ value for an independent MC sampling run using the same input conformer energies. The slope of the graph gives the $-\Delta \mathrm{S}$ of $0.0045 \mathrm{kcal} / \mathrm{mol} /{ }^{\circ}$ and intercept gives the $\Delta \mathrm{H}$ of $0.387 \mathrm{kcal} / \mathrm{mol}, \mathrm{R}^{2}$ is 0.979. (B) Van't Hoff plot for the same dataset. The slope is $\Delta H / R$ and intercept $\Delta S / R$ where $\mathrm{R}$ is the gas constant. $\Delta \mathrm{H}$ is $0.377 \mathrm{kcal} / \mathrm{mol}$ and $-\Delta \mathrm{S}$ is $0.0045 \mathrm{kcal} / \mathrm{mol}$. 
Correlation analysis of protonation states of residues in RCs. A major advantage of having access to the microstates it that the correlation between conformer choices can be found. Here we will describe the correlation of residue protonation states in different microstates using the much larger RCs as an example. It has 828 residues in the coordinate file. There are 132 ionizable residues (Asp, Glu, Arg, Lys and His) and NTR, CTR, and two redox active quinones and other cofactors. There are 1743 isosteric conformers for the protein, cofactors and retained water molecules. More than 45 residues are found in a mixture of ionization states at $\mathrm{pH} 7$ and there are more than 50,000 unique protonation microstates to investigate (Table 1).

RCs show the distinction between the lowest energy microstates and those with highest probability. We determined the lowest energy example of each of the thirty most probable protonation microstates (Figure S4). These were compared with the ranking of the states at lowest energy. While the high probability states are enriched with lower energy microstates they are not identical. This shows that the conformational degrees of freedom can create more opportunities to make some protonation microstates than others.

In this system the proton distribution is functionally important as a proton must be brought into the $\mathrm{Q}_{\text {в }}$ site, $15 \AA$ from the surface, through a network of protonatable residues and waters. ${ }^{63-}$ 65 The Pearson weighted correlation coefficient is used to find the correlation between the residues (equation 3). Residues whose charge state is the same in all microstates are removed from the analysis, as these are not correlated with any other. The weighted correlation coefficient is obtained by taking the unique charge microstates with its corresponding count for the $\approx 45$ remaining residues. Only the residues that have an absolute value of the correlation of at least 0.1 with any other residue are included in the heatmap (Figure 5). This procedure identifies 15-16 residues, all of which are near the $Q_{B}$ site.
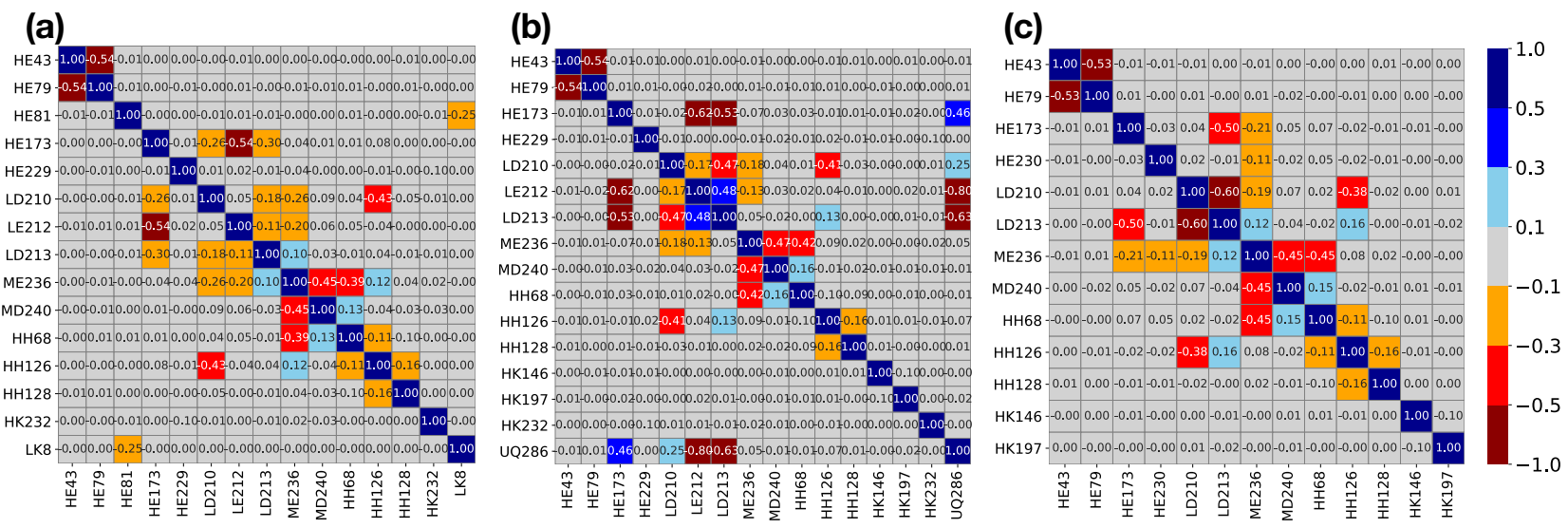

Figure 5. Heat map of Pearson's weighted correlation coefficient of residue ionization states in RCs at pH 7. Only residues with an absolute value for the correlation $\geq 0.1$ with at least one other residue are shown. Each square in the heat map gives the correlation strength for the two residues obtained with equation 3 . Residue are identified as: chain ( $\mathrm{L}, \mathrm{M}$ or $\mathrm{H})$, one letter residue name, then residue number. Ubiquinone is UQ. Dark blue, blue, sky blue, light gray, orange, red and dark red correspond to the correlation value of range 0.5 to $1.0,0.3$ to $0.5,0.1$ to $0.3,-0.1$ to $0.1,-0.3$ to $-0.1,-0.5$ to -0.3 and -1.0 to -0.5 respectively. (a) Neutral quinone (b) 50:50 mixture of two state (c) Anionic semiquinone $\mathrm{Q}_{\mathrm{B}}{ }^{\circ}$. The quinone is not seen in panel a or $\mathrm{c}$ as it is $100 \%$ in a single redox state.

The correlation analysis carried out for the $\mathrm{RC}$ protonation microstate distribution in three quinone states: neutral quinone, $\approx 50: 50$ mixture of the two redox states and the anionic semiquinone $\mathrm{QB}^{\bullet^{\bullet}}$. A positive correlation indicates that the two groups are more likely to be ionized together, while 
negative correlation shows that ionization of one reduces ionization of the other. Thus, in the

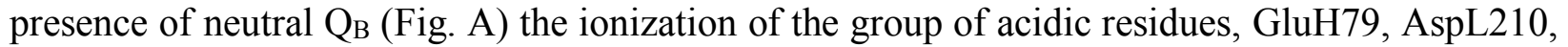
GluL212 and AspL213 are negatively correlated with each other. Ionization of AspM240 and HisH68 are positively correlated. In the presence of $\mathrm{Q}_{\mathrm{B}}{ }^{\bullet-}$ the heat map has two fewer residues than

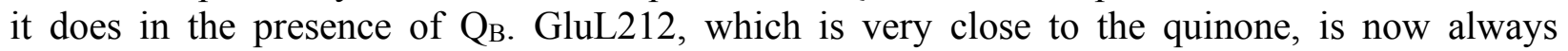
protonated. ${ }^{7}$ As the quinone redox state is expected to modify the protonation states in its vicinity, a heat map is prepared at an $\mathrm{E}_{\mathrm{h}}$ where the quinone is $\approx 50 \%$ reduced in the MCCE MC sampling. The protonation of residues that are correlated with the quinone redox state can be seen. Thus, GluL212 and GluL213 are less ionized when the quinone is reduced, while GluH173 and AspL210 are more ionized. Figure 6 shows the position of the residues that are interacting with each other.

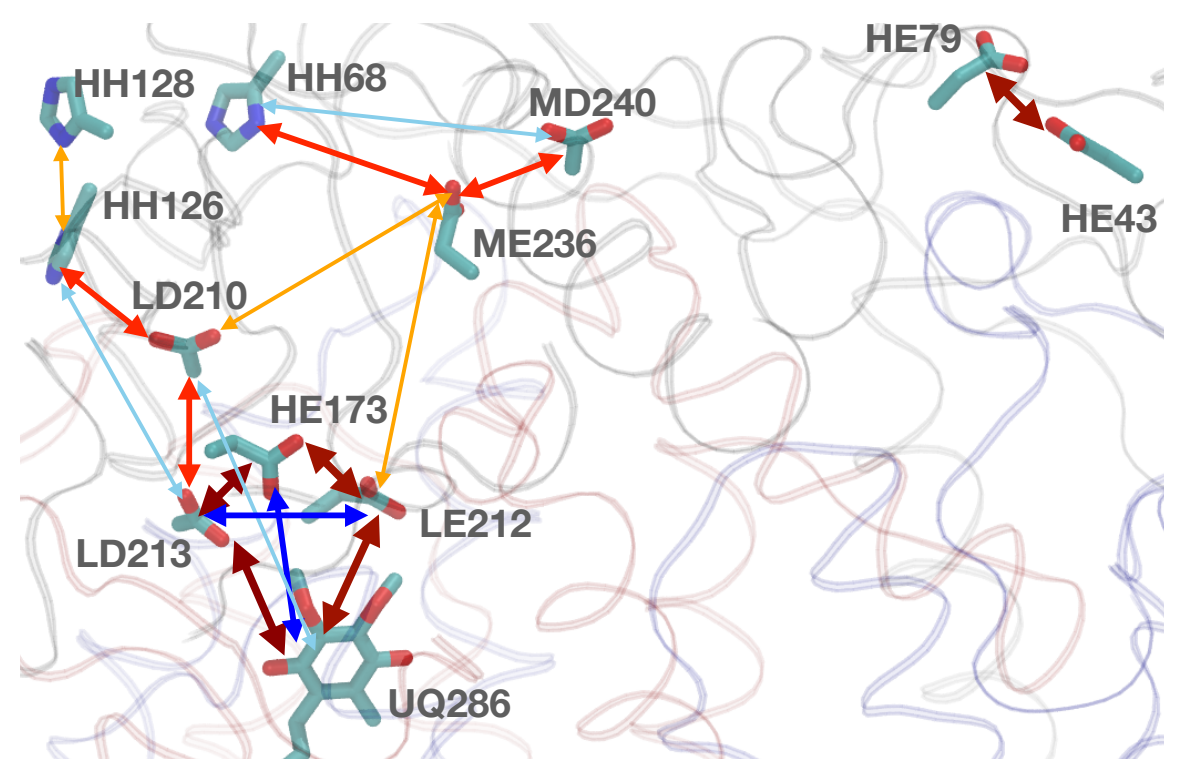

Figure 6. Structure representation of residues that show correlations in the MC sampling with a 50:50 mixture of $\mathrm{Q}_{\mathrm{B}}$ and $\mathrm{Q}_{\mathrm{B}}{ }^{-}$. Only those residues whose absolute correlation coefficient is $\geq 0.1$ are shown. Blue and red two-sided arrows show the positive and negative correlation respectively. The color code is same as Figure 5. Wider arrows indicate higher correlation.

Other uses of microstate analysis in MCCE. There are other important properties of proteins whose analysis relies on knowing the microstates rather than simply the average properties. The correlation of structures and protonation state can show how small changes influence the proton affinity of interior sites in the protein. ${ }^{66}$ In proton pumps these changes can lead to efficient proton loading and unloading as protons are transferred through the protein. ${ }^{12,67}$ Microstate analysis can show how the protonation states of ligands can shift those of the protein. The proton transfer paths require continuous hydrogen bonded connections. ${ }^{68,69} \mathrm{MCCE}$ has been used to identify the hydrogen bonds in the MC ensemble to trace proton transfer paths. ${ }^{42,70-72}$ High probability or low energy microstates that define residue protonation and neutral His tautomers provide rational input to standard MD. 


\section{CONCLUSIONS}

Proteins have many acidic and basic residues and are very unlikely to be in a single protonation state. Microstate analysis allows us to characterize the ensemble of protonation microstates. This shows proteins have a range of charge states and for each charge state that there are multiple tautomers with different distribution of protons. While there can be an astonishing number of protonation microstates found in proteins such as RCs only a few have significant probability. The lower population states may play roles as intermediates in important processes such as proton transfers. The microstate analysis shows how the protonation state of groups of residues are coupled together and which residues are not correlated with each other. Analysis, not carried out here, can show how individual hydrogen bonding patterns can stabilize particular protonation states or be aligned for proton transport. The $\mathrm{MC}$ ensemble is large enough that calculations of the thermodynamic parameters including the $\Delta \mathrm{S}$ of proton binding can be calculated with reasonable precision.

\section{ACKNOWLEDGMENTS}

The authors would like to acknowledge the funds from the National Science Foundation grant MCB-1519640.

\section{REFERENCES}

(1) Intlekofer, A. M.; Wang, B.; Liu, H.; Shah, H.; Carmona-Fontaine, C.; Rustenburg, A. S.; Salah, S.; Gunner, M. R.; Chodera, J. D.; Cross, J. R.; Thompson, C. B. L-2-Hydroxyglutarate Production Arises from Noncanonical Enzyme Function at Acidic PH. Nat. Chem. Biol. 2017, 13 (5), 494-500. https://doi.org/10.1038/nchembio.2307.

(2) Czodrowski, P.; Sotriffer, C. A.; Klebe, G. Protonation Changes upon Ligand Binding to Trypsin and Thrombin: Structural Interpretation Based on PK(a) Calculations and ITC Experiments. J. Mol. Biol. 2007, 367 (5), 1347-1356.

https://doi.org/10.1016/j.jmb.2007.01.022.

(3) Czodrowski, P.; Sotriffer, C. A.; Klebe, G. Atypical Protonation States in the Active Site of HIV-1 Protease: A Computational Study. J. Chem. Inf. Model. 2007, 47 (4), 1590-1598. https://doi.org/10.1021/ci600522c.

(4) Song, Y.; Gunner, M. R. Using Multiconformation Continuum Electrostatics to Compare Chloride Binding Motifs in a-Amylase, Human Serum Albumin, and Omp32. J. Mol. Biol. 2009, 387 (4), 840-856. https://doi.org/10.1016/j.jmb.2009.01.038.

(5) Aguilar, B.; Anandakrishnan, R.; Ruscio, J. Z.; Onufriev, A. V. Statistics and Physical Origins of PK and Ionization State Changes upon Protein-Ligand Binding. Biophys. J. 2010, 98 (5), 872-880. https://doi.org/10.1016/j.bpj.2009.11.016.

(6) Kim, J.; Mao, J.; Gunner, M. R. Are Acidic and Basic Groups in Buried Proteins Predicted to Be lonized? J. Mol. Biol. 2005, 348 (5), 1283-1298. https://doi.org/10.1016/j.jmb.2005.03.051.

(7) Okamura, M. Y.; Paddock, M. L.; Graige, M. S.; Feher, G. Proton and Electron Transfer in Bacterial Reaction Centers. Biochim. Biophys. Acta 2000, 1458 (1), 148-163. https://doi.org/10.1016/S0005-2728(00)00065-7. 
(8) Wraight, C. A. Chance and Design-Proton Transfer in Water, Channels and Bioenergetic Proteins. Biochim. Biophys. Acta 2006, 1757 (8), 886-912.

https://doi.org/10.1016/j.bbabio.2006.06.017.

(9) Gunner, M. R.; Amin, M.; Zhu, X.; Lu, J. Molecular Mechanisms for Generating Transmembrane Proton Gradients. Biochim. Biophys. Acta 2013, 1827 (8-9), 892-913. https://doi.org/10.1016/j.bbabio.2013.03.001.

(10) Ishikita, H.; Saito, K. Proton Transfer Reactions and Hydrogen-Bond Networks in Protein Environments. J. Royal Soc. Interface 2013, 11 (91), $20130518-20130518$. https://doi.org/10.1098/rsif.2013.0518.

(11) Wikström, M.; Sharma, V.; Kaila, V. R. I.; Hosler, J. P.; Hummer, G. New Perspectives on Proton Pumping in Cellular Respiration. Chem. Rev. 2015, 115 (5), 2196-2221. https://doi.org/10.1021/cr500448t.

(12) Cai, X.; Son, C. Y.; Mao, J.; Kaur, D.; Zhang, Y.; Khaniya, U.; Cui, Q.; Gunner, M. R. Identifying the Proton Loading Site Cluster in the Ba3 Cytochrome c Oxidase That Loads and Traps Protons. Biochim. Biophys. Acta 2020, 1861 (10), 148239. https://doi.org/10.1016/j.bbabio.2020.148239.

(13) Kaur, D.; Khaniya, U.; Zhang, Y.; Gunner, M. R. Protein Motifs for Proton Transfers That Build the Transmembrane Proton Gradient. Front. Chem. 2021, 9, 446. https://doi.org/10.3389/fchem.2021.660954.

(14) Shao, J.; Tanner, S. W.; Thompson, N.; Cheatham, T. E. Clustering Molecular Dynamics Trajectories: 1. Characterizing the Performance of Different Clustering Algorithms. J. Chem. Theory Comput. 2007, 3 (6), 2312-2334. https://doi.org/10.1021/ct700119m.

(15) Husic, B. E.; Pande, V. S. Ward Clustering Improves Cross-Validated Markov State Models of Protein Folding. J. Chem. Theory Comput. 2017, 13 (3), 963-967. https://doi.org/10.1021/acs.jctc.6b01238.

(16) Haack, F.; Fackeldey, K.; Röblitz, S.; Scharkoi, O.; Weber, M.; Schmidt, B. Adaptive Spectral Clustering with Application to Tripeptide Conformation Analysis. J. Chem. Phys. 2013, 139 (19), 194110. https://doi.org/10.1063/1.4830409.

(17) Song, Y.; Mao, J.; Gunner, M. R. MCCE2: Improving Protein PKa Calculations with Extensive Side Chain Rotamer Sampling. J. Comput. Chem. 2009, 30 (14), 2231-2247. https://doi.org/10.1002/jcc.21222.

(18) Citra, M. J. Estimating the $\mathrm{PK}_{\mathrm{a}}$ of Phenols, Carboxylic Acids and Alcohols from SemiEmpirical Quantum Chemical Methods. Chemosphere 1999, 38 (1), 191-206. https://doi.org/10.1016/S0045-6535(98)00172-6.

(19) Tehan, B. G.; Lloyd, E. J.; Wong, M. G.; Pitt, W. R.; Montana, J. G.; Manallack, D. T.; Gancia, E. Estimation of $\mathrm{PK}_{\mathrm{a}}$ Using Semiempirical Molecular Orbital Methods. Part 1: Application to Phenols and Carboxylic Acids. Quant. Struct-Act. Rel. 2002, 21 (5), 457472. https://doi.org/10.1002/1521-3838(200211)21:5<457::AID-QSAR457>3.0.CO;2-5.

(20) Baker, N. A. Improving Implicit Solvent Simulations: A Poisson-Centric View. Curr. Opin. Struct. Biol. 2005, 15 (2), 137-143. https://doi.org/10.1016/j.sbi.2005.02.001.

(21) Gunner, M. R.; Baker, N. A. Continuum Electrostatics Approaches to Calculating PK $\mathrm{K}_{\mathrm{a}} \mathrm{S}$ and $\mathrm{E}_{\mathrm{m}} \mathrm{s}$ in Proteins. Meth. Enzymol. 2016, 578, 1-20. https://doi.org/10.1016/bs.mie.2016.05.052. 
(22) Alexov, E.; Mehler, E. L.; Baker, N.; Baptista, A.; Huang, Y.; Milletti, F.; Nielsen, J. E.; Farrell, D.; Carstensen, T.; Olsson, M. H. M.; Shen, J. K.; Warwicker, J.; Williams, S.; Word, J. M. Progress in the Prediction of $\mathrm{PK}_{a}$ Values in Proteins. Proteins 2011, 79 (12), 32603275. https://doi.org/10.1002/prot.23189.

(23) Baptista, A. M.; Martel, P. J.; Petersen, S. B. Simulation of Protein Conformational Freedom as a Function of $\mathrm{PH}$ : Constant-PH Molecular Dynamics Using Implicit Titration. Proteins 1997, 27 (4), 523-544.

(24) Wallace, J. A.; Shen, J. K. Predicting $\mathrm{PK}_{\mathrm{a}}$ Values with Continuous Constant PH Molecular Dynamics. Methods Enzymol. 2009, 466, 455-475. https://doi.org/10.1016/S00766879(09)66019-5.

(25) Swails, J. M.; York, D. M.; Roitberg, A. E. Constant PH Replica Exchange Molecular Dynamics in Explicit Solvent Using Discrete Protonation States: Implementation, Testing, and Validation. J. Chem. Theory Comput. 2014, 10 (3), 1341-1352.

https://doi.org/10.1021/ct401042b.

(26) Radak, B. K.; Chipot, C.; Suh, D.; Jo, S.; Jiang, W.; Phillips, J. C.; Schulten, K.; Roux, B. Constant-PH Molecular Dynamics Simulations for Large Biomolecular Systems. J. Chem. Theory Comput. 2017, 13 (12), 5933-5944. https://doi.org/10.1021/acs.jctc.7b00875.

(27) Damjanovic, A.; Miller, B. T.; Okur, A.; Brooks, B. R. Reservoir PH Replica Exchange. J. Chem. Phys. 2018, 149 (7), 072321. https://doi.org/10.1063/1.5027413.

(28) Aleksandrov, A.; Roux, B.; MacKerell, A. D. PKa Calculations with the Polarizable Drude Force Field and Poisson-Boltzmann Solvation Model. J. Chem. Theory Comput. 2020, 16 (7), 4655-4668. https://doi.org/10.1021/acs.jctc.0c00111.

(29) Olsson, M. H. M.; Søndergaard, C. R.; Rostkowski, M.; Jensen, J. H. PROPKA3: Consistent Treatment of Internal and Surface Residues in Empirical PK $\mathrm{K}_{\mathrm{a}}$ Predictions. J. Chem. Theory Comput. 2011, 7 (2), 525-537. https://doi.org/10.1021/ct100578z.

(30) Wang, L.; Zhang, M.; Alexov, E. DelPhiPKa Web Server: Predicting PK $\mathrm{K}_{\mathrm{a}}$ of Proteins, RNAs and DNAs. Bioinformatics 2016, 32 (4), 614-615. https://doi.org/10.1093/bioinformatics/btv607.

(31) Anandakrishnan, R.; Aguilar, B.; Onufriev, A. V. H++ 3.0: Automating PK Prediction and the Preparation of Biomolecular Structures for Atomistic Molecular Modeling and Simulations. Nucleic Acids Res. 2012, 40 (Web Server issue), W537-541. https://doi.org/10.1093/nar/gks375.

(32) Reis, P. B. P. S.; Vila-Viçosa, D.; Rocchia, W.; Machuqueiro, M. PypKa: A Flexible Python Module for Poisson-Boltzmann-Based PKa Calculations. J. Chem. Inf. Model. 2020. https://doi.org/10.1021/acs.jcim.0c00718.

(33) Kuramitsu, S.; Hamaguchi, K. Analysis of the Acid-Base Titration Curve of Hen Lysozyme. J Biochem 1980, 87 (4), 1215-1219.

(34) Bartik, K.; Redfield, C.; Dobson, C. M. Measurement of the Individual PKa Values of Acidic Residues of Hen and Turkey Lysozymes by Two-Dimensional 1H NMR. Biophys. J. 1994, 66 (4), 1180-1184.

(35) Alexov, E. G.; Gunner, M. R. Incorporating Protein Conformational Flexibility into the Calculation of PH-Dependent Protein Properties. Biophys. J. 1997, 72 (5), 2075-2093. 
(36) Kuehner, D. E.; Engmann, J.; Fergg, F.; Wernick, M.; Blanch, H. W.; Prausnitz, J. M. Lysozyme Net Charge and Ion Binding in Concentrated Aqueous Electrolyte Solutions. J. Phys. Chem. B 1999, 103 (8), 1368-1374. https://doi.org/10.1021/jp983852i.

(37) Paddock, M. L.; Rongey, S. H.; McPherson, P. H.; Juth, A.; Feher, G.; Okamura, M. Y. Pathway of Proton Transfer in Bacterial Reaction Centers: Role of Aspartate-L213 in Proton Transfers Associated with Reduction of Quinoneto Dihydroquinone. Biochemistry 1994, 33 (3), 734-745. https://doi.org/10.1021/bi00169a015.

(38) Alexov, E.; Miksovska, J.; Baciou, L.; Schiffer, M.; Hanson, D. K.; Sebban, P.; Gunner, M. R. Modeling the Effects of Mutations on the Free Energy of the First Electron Transfer from $Q_{A}{ }^{-}$to $Q_{B}$ in Photosynthetic Reaction Centers. Biochemistry 2000, 39 (20), 5940-5952. https://doi.org/10.1021/bi9929498.

(39) Walsh, M. A.; Schneider, T. R.; Sieker, L. C.; Dauter, Z.; Lamzin, V. S.; Wilson, K. S. Refinement of Triclinic Hen Egg-White Lysozyme at Atomic Resolution. Acta Crystallogr. A 1998, 54 (4), 522-546. https://doi.org/10.1107/S0907444997013656.

(40) Stowell, M. H.; McPhillips, T. M.; Rees, D. C.; Soltis, S. M.; Abresch, E.; Feher, G. LightInduced Structural Changes in Photosynthetic Reaction Center: Implications for Mechanism of Electron-Proton Transfer. Science 1997, 276 (5313), 812-816. https://doi.org/10.1126/science.276.5313.812.

(41) Gunner, M. R.; Zhu, X.; Klein, M. C. MCCE Analysis of the PK ${ }_{a}$ s of Introduced Buried Acids and Bases in Staphylococcal Nuclease. Proteins 2011, 79 (12), 3306-3319. https://doi.org/10.1002/prot.23124.

(42) Zhang, Y.; Haider, K.; Kaur, D.; Ngo, V. A.; Cai, X.; Mao, J.; Khaniya, U.; Zhu, X.; Noskov, S.; Lazaridis, T.; Gunner, M. R. Characterizing the Water Wire in the Gramicidin Channel Found by Monte Carlo Sampling Using Continuum Electrostatics and in Molecular Dynamics Trajectories with Conventional or Polarizable Force Fields. J. Comput. Biophys. Chem. 2021, 20 (02), 111-130. https://doi.org/10.1142/S2737416520420016.

(43) Warwicker, J.; Watson, H. C. Calculation of the Electric Potential in the Active Site Cleft Due to $\alpha$-Helix Dipoles. J. Mol. Biol. 1982, 157 (4), 671-679. https://doi.org/10.1016/0022-2836(82)90505-8.

(44) Rocchia, W.; Alexov, E.; Honig, B. Extending the Applicability of the Nonlinear Poisson-Boltzmann Equation: Multiple Dielectric Constants and Multivalent Ions. J. Phys. Chem. B 2001, 105 (28), 6507-6514. https://doi.org/10.1021/jp010454y.

(45) Sitkoff, D.; Sharp, K. A.; Honig, B. Accurate Calculation of Hydration Free Energies Using Macroscopic Solvent Models. J. Phys. Chem. 1994, 98 (7), 1978-1988. https://doi.org/10.1021/j100058a043.

(46) Cornell, W. D.; Cieplak, P.; Bayly, C. I.; Gould, I. R.; Merz, K. M.; Ferguson, D. M.; Spellmeyer, D. C.; Fox, T.; Caldwell, J. W.; Kollman, P. A. A Second Generation Force Field for the Simulation of Proteins, Nucleic Acids, and Organic Molecules. J. Am. Chem. Soc. 1995, 117 (19), 5179-5197. https://doi.org/10.1021/ja00124a002.

(47) Beroza, P.; Fredkin, D. R.; Okamura, M. Y.; Feher, G. Protonation of Interacting Residues in a Protein by a Monte Carlo Method: Application to Lysozyme and the Photosynthetic Reaction Center of Rhodobacter Sphaeroides. Proc. Natl. Acad. Sci. U.S.A. 1991, 88 (13), 5804-5808. 
(48) Yang, A. S.; Gunner, M. R.; Sampogna, R.; Sharp, K.; Honig, B. On the Calculation of PKas in Proteins. Proteins 1993, 15 (3), 252-265. https://doi.org/10.1002/prot.340150304.

(49) Liu, Y.; Meng, Q.; Chen, R.; Wang, J.; Jiang, S.; Hu, Y. A New Method to Evaluate the Similarity of Chromatographic Fingerprints: Weighted Pearson Product-Moment Correlation Coefficient. J. Chromatogr. Sci. 2004, 42 (10), 545-550. https://doi.org/10.1093/chromsci/42.10.545.

(50) Frick, D. N.; Rypma, R. S.; Lam, A. M. I.; Frenz, C. M. Electrostatic Analysis of the Hepatitis C Virus NS3 Helicase Reveals Both Active and Allosteric Site Locations. Nucleic Acids Res. 2004, 32 (18), 5519-5528. https://doi.org/10.1093/nar/gkh891.

(51) Hong, J.; Hamers, R. J.; Pedersen, J. A.; Cui, Q. A Hybrid Molecular Dynamics/Multiconformer Continuum Electrostatics (MD/MCCE) Approach for the Determination of Surface Charge of Nanomaterials. J. Phys. Chem. C 2017, 121 (6), 35843596. https://doi.org/10.1021/acs.jpcc.6b11537.

(52) Durant, J. A.; Chen, C.; Laue, T. M.; Moody, T. P.; Allison, S. A. Use of T4 Lysozyme Charge Mutants to Examine Electrophoretic Models. Biophys. Chem. 2002, 101-102, 593-609. https://doi.org/10.1016/s0301-4622(02)00168-0.

(53) Moody, T. P.; Kingsbury, J. S.; Durant, J. A.; Wilson, T. J.; Chase, S. F.; Laue, T. M. Valence and Anion Binding of Bovine Ribonuclease A between PH 6 and 8. Anal. Biochem. 2005, 336 (2), 243-252. https://doi.org/10.1016/j.ab.2004.09.009.

(54) Laue, T. Charge Matters. Biophys. Rev. 2016, 8 (4), 287-289. https://doi.org/10.1007/s12551-016-0229-3.

(55) Levy, R. M.; Gallicchio, E. Computer Simulations with Explicit Solvent: Recent Progress in the Thermodynamic Decomposition of Free Energies and in Modeling Electrostatic Effects. Annu. Rev. Phys. Chem. 1998, 49 (1), 531-567. https://doi.org/10.1146/annurev.physchem.49.1.531.

(56) Ahmad, M.; Helms, V.; Lengauer, T.; Kalinina, O. V. Enthalpy-Entropy Compensation upon Molecular Conformational Changes. J. Chem. Theory Comput. 2015, 11 (4), 1410-1418. https://doi.org/10.1021/ct501161t.

(57) Chen, L.; Cruz, A.; Roe, D. R.; Simmonett, A. C.; Wickstrom, L.; Deng, N.; Kurtzman, T. Thermodynamic Decomposition of Solvation Free Energies with Particle Mesh Ewald and Long-Range Lennard-Jones Interactions in Grid Inhomogeneous Solvation Theory. J.

Chem. Theory Comput. 2021, 17 (5), 2714-2724. https://doi.org/10.1021/acs.jctc.0c01185.

(58) Peter, C.; Oostenbrink, C.; van Dorp, A.; van Gunsteren, W. F. Estimating Entropies from Molecular Dynamics Simulations. J. Chem. Phys. 2004, 120 (6), 2652-2661. https://doi.org/10.1063/1.1636153.

(59) Zhukov, A.; Karlsson, R. Statistical Aspects of van't Hoff Analysis: A Simulation Study. J. Mol. Recognit. 2007, 20 (5), 379-385. https://doi.org/10.1002/jmr.845.

(60) Ross, G. A.; Bodnarchuk, M. S.; Essex, J. W. Water Sites, Networks, and Free Energies with Grand Canonical Monte Carlo. J. Am. Chem. Soc. 2015, 137 (47), 14930-14943. https://doi.org/10.1021/jacs.5b07940.

(61) Ross, G. A.; Bruce Macdonald, H. E.; Cave-Ayland, C.; Cabedo Martinez, A. I.; Essex, J. W. Replica-Exchange and Standard State Binding Free Energies with Grand Canonical Monte 
Carlo. J. Chem. Theory Comput. 2017, 13 (12), 6373-6381.

https://doi.org/10.1021/acs.jctc.7b00738.

(62) Khrapunov, S. The Enthalpy-Entropy Compensation Phenomenon. Limitations for the Use of Some Basic Thermodynamic Equations. Curr. Protein Pept. Sci. 2018, 19 (11), 10881091. https://doi.org/10.2174/1389203719666180521092615.

(63) Okamura, M. Y.; Feher, G. Proton Transfer in Reaction Centers from Photosynthetic Bacteria. Annu. Rev. Biochem. 1992, 61, 861-896. https://doi.org/10.1146/annurev.bi.61.070192.004241.

(64) Abresch, E. C.; Paddock, M. L.; Stowell, M. H. B.; McPhillips, T. M.; Axelrod, H. L.; Soltis, S. M.; Rees, D. C.; Okamura, M. Y.; Feher, G. Identification of Proton Transfer Pathways in the X-Ray Crystal Structure of the Bacterial Reaction Center from Rhodobacter Sphaeroides. Photosynth. Res. 1998, 55 (2), 119-125. https://doi.org/10.1023/A:1006047519260.

(65) Krammer, E.-M.; Till, M. S.; Sebban, P.; Ullmann, G. M. Proton-Transfer Pathways in Photosynthetic Reaction Centers Analyzed by Profile Hidden Markov Models and Network Calculations. J. Mol. Biol. 2009, 388 (3), 631-643. https://doi.org/10.1016/j.jmb.2009.03.020.

(66) Di Luca, A.; Gamiz-Hernandez, A. P.; Kaila, V. R. I. Symmetry-Related Proton Transfer Pathways in Respiratory Complex I. Proc. Natl. Acad. Sci. U.S.A. 2017, 114 (31), E6314E6321. https://doi.org/10.1073/pnas.1706278114.

(67) Chenal, C.; Gunner, M. R. Two Cl lons and a Glu Compete for a Helix Cage in the CLC Proton/Cl- Antiporter. Biophys. J. 2017, 113 (5), 1025-1036. https://doi.org/10.1016/j.bpj.2017.07.025.

(68) Miyake, T.; Rolandi, M. Grotthuss Mechanisms: From Proton Transport in Proton Wires to Bioprotonic Devices. J. Phys.: Condens. Matter 2015, 28 (2), 023001. https://doi.org/10.1088/0953-8984/28/2/023001.

(69) Siemers, M.; Lazaratos, M.; Karathanou, K.; Guerra, F.; Brown, L. S.; Bondar, A.-N. Bridge: A Graph-Based Algorithm to Analyze Dynamic H-Bond Networks in Membrane Proteins. J. Chem. Theory Comput. 2019, 15 (12), 6781-6798. https://doi.org/10.1021/acs.jctc.9b00697.

(70) Cai, X.; Haider, K.; Lu, J.; Radic, S.; Son, C. Y.; Cui, Q.; Gunner, M. R. Network Analysis of a Proposed Exit Pathway for Protons to the P-Side of Cytochrome c Oxidase. Biochim. Biophys. Acta 2018, 1859 (10), 997-1005. https://doi.org/10.1016/j.bbabio.2018.05.010.

(71) Khaniya, U.; Gupta, C.; Cai, X.; Mao, J.; Kaur, D.; Zhang, Y.; Singharoy, A.; Gunner, M. R. Hydrogen Bond Network Analysis Reveals the Pathway for the Proton Transfer in the EChannel of T. Thermophilus Complex I. Biochim. Biophys. Acta 2020, 1861 (10), 148240. https://doi.org/10.1016/j.bbabio.2020.148240.

(72) Kaur, D.; Zhang, Y.; Reiss, K. M.; Mandal, M.; Brudvig, G. W.; Batista, V. S.; Gunner, M. R. Proton Exit Pathways Surrounding the Oxygen Evolving Complex of Photosystem II. Biochim. Biophys. Acta 2021, 1862 (8), 148446. https://doi.org/10.1016/j.bbabio.2021.148446. 
bioRxiv preprint doi: https://doi.org/10.1101/2022.01.07.475457; this version posted January 10,2022 . The copyright holder for this preprint (which was not certified by peer review) is the author/funder, who has granted bioRxiv a license to display the preprint in perpetuity. It is made available under aCC-BY-NC-ND 4.0 International license.

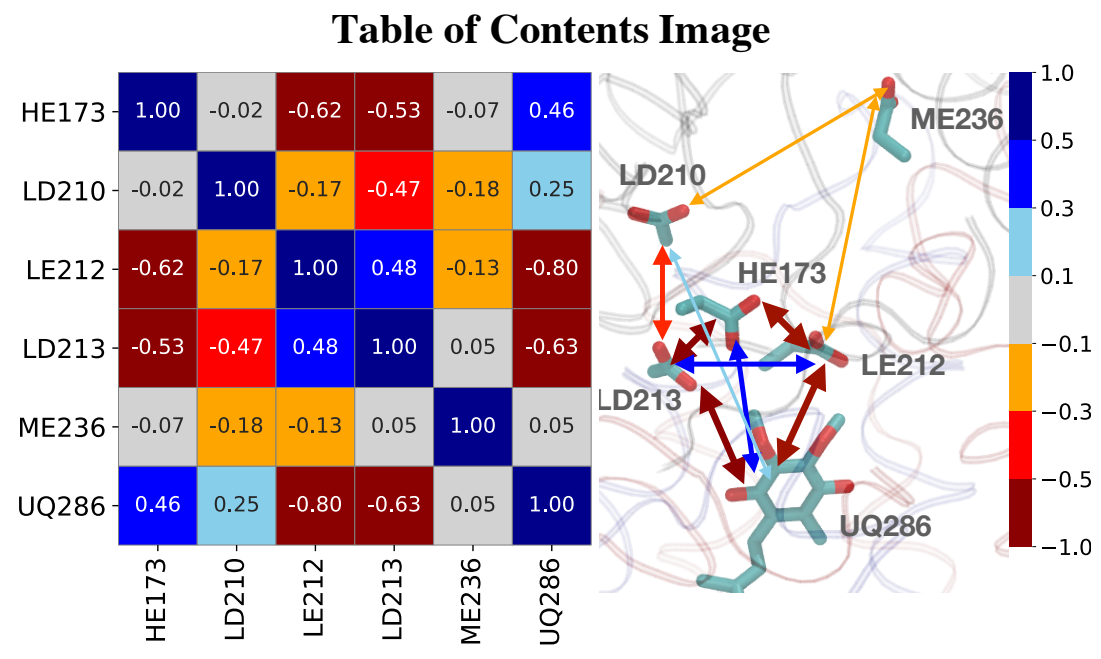

Figure: Table of content image 